\title{
Enhanced Supercontinuum Generation By Minute Continuous Wave Seed
}

\author{
Kim K. Y. Cheung, Chi Zhang, Yue Zhou, Kenneth K. Y. Wong and Kevin K. Tsia*, \\ Department of Electrical and Electronic Engineering, The University of Hong Kong, Pokfulam Road, \\ Hong Kong;
}

\begin{abstract}
It is well-known that the properties of the supercontinuum (SC) radiation depend critically on the initial pumping conditions. For instance, if the SC is initiated by noise, namely modulation instability (MI), statistically rare "rogue waves" can be observed with sizable spectral broadening and intensity enhancement in SC. Interestingly, such rouge events can be actively controlled by adding an external weak pulse or by modulating the pump-pulse envelopes. In contrast, we here present that a simple triggering mechanism using an extremely weak continuous wave (CW) can also achieve such "rogue" enhancement. We experimentally demonstrated that a weak CW trigger $(\sim 200,000 \times$ weaker than pump) can considerably broaden the SC bandwidth compared to the untriggered SC case ( 100 nm wider). Such enhancement was found to occur when the $\mathrm{CW}$ trigger's wavelength falls roughly within the modulation instability gain bandwidth. CW triggering also significantly alters the SC amplitude statistics, i.e. from extreme-value statistics in the untriggered SC to almost normal distribution in the triggered SC. Interferometric measurements also revealed the improvement in the $\mathrm{SC}$ coherence when the $\mathrm{SC}$ is $\mathrm{CW}$-triggered. The enhanced $\mathrm{SC}$ by minute $\mathrm{CW}$ triggering only requires the $\mathrm{CW}$-wavelength tuning for optimization and eliminates the necessity of high-precision (down to picoseconds) timing between the pump and the seed as in the pulse-seeding case. It thus offers a more convenient and practical approach to realize an enhanced and stable SC for many applications, especially in which real-time, ultrafast and singleshot spectroscopic measurements are essential.
\end{abstract}

Keywords: Nonlinear optics, Supercontinuum generation

\section{INTRODUCTION}

As the salient field in nonlinear optics, supercontinuum (SC) radiation, which signifies an ultra-wideband spectrum, represents a ubiquitous light source for a myriad of applications, including frequency metrology, optical coherence tomography, molecular optical spectroscopy (e.g. fluorescence, absorption and vibrational spectroscopy), optical coherence tomography, and wavelength-division multiplexing (WDM) optical communications. The extensive spectral broadening in SC involves complex interactions among manifold optical nonlinear effects, each of which depends remarkably on many different parameters, namely the input light power and temporal pulse width, the fiber's nonlinearity and group velocity dispersion (GVD) and so on [1]. By far, a mechanism based on soliton-fission initiated by modulation-instability (MI) is one of the most effective approaches to generate ultrabroadband SC [2]. This process involves MI which creates spectral sidebands to the narrowband input pump light to an extent that Raman-induced selffrequency shift can break it into multiple red-shifted solitons. As MI grows spontaneously from noise, the subsequent soliton fission is triggered in a random fashion. Consequently, the SC generated by this approach is inherently incoherent and experiences significant pulse-to-pulse amplitude fluctuation [3]. In its extreme case, optical rogue waves can even be observed below the "threshold" for soliton fission [4]. Such degradation of SC stability, albeit the SC possesses an ultrabroadband spectrum, impedes the utility of SC in many practical applications, particularly where measurements of rapid dynamics and non-repetitive transient processes are essential, for instance, real-time, ultrafast and single-shot spectroscopy [5] and microscopy [6].

Fortunately, such random and extreme SC instability can be manipulated by adding an external weak pulse copropagating with the pump light pulse [7] or by modulating the pump pulse envelopes [8]. These schemes show that such active controls can lead to drastic change in the SC's noise characteristics, especially are able to deliberately "stimulate"

Nonlinear Frequency Generation and Conversion: Materials, Devices, and Applications X, edited by Konstantin L. Vodopyanov, Proc. of SPIE Vol. 7917, 79170X · C 2011 SPIE

CCC code: $0277-786 \mathrm{X} / 11 / \$ 18 \cdot$ doi: $10.1117 / 12.873137$

Proc. of SPIE Vol. 7917 79170X-1 
the rogue events in the SC [7]. We here present that a simple triggering mechanism using a separate, extremely weak continuous wave $(\mathrm{CW})$ can also achieve such rogue enhancement and control the SC instability. By launching a CW $\mathrm{CW}$ trigger light ( 200,000× weaker than pump) at a wavelength within the MI gain bandwidth together with the pump light, the MI growth process can be then initiated by such controllable CW trigger, rather than noise. Hence, the SC generation can be greatly enhanced in terms of both power and stability. We observed that introducing such minute $\mathrm{CW}$ trigger can considerably broaden the SC bandwidth $(\sim 100 \mathrm{~nm}$ wider), with more than 100 -fold higher in average SC power compared to the untriggered SC case. . The $\mathrm{CW}$ triggering also significantly alters the SC amplitude statistics, i.e. from the extreme-value statistics in the untriggered SC to the almost Gaussian distribution in the CW-triggered SC. Interferometric measurements further revealed the improvement in the coherence of the triggered SC in contrast to the untriggered one.

We note that SC coherence was shown to increase considerably as the pump pulse width is progressively narrower $(<50 \mathrm{fs})$. However, such ultra-short light pulses are drastically sensitive to ambient perturbation and hence are not practical in many applications. In addition, specialty dispersion-engineered fibers have also been demonstrated to be useful for reducing excess noise for SC generation. But the effect is static, not dynamically controllable. To this end, CW triggering offers a handy approach to provide a great degree of active control over the SC generation process and can also benefit the longer-pulse ( $>$ ps) pumping scheme to generate a more stable and enhanced SC - expanding the scope of possibilities in SC generation. For instance, the longer pulse excitation ( $\mathrm{ps}-\mathrm{ns}$ ) would be more desirable for the majority of the fluorescence and nonlinear optical microscopy techniques $[9,10]$. The present CW triggering scheme mainly only requires the $\mathrm{CW}$-wavelength tuning for optimization and eliminates the necessity of high-precision (down to picoseconds) timing between the pump and seed as in the pulse-seeding case [7]. It thus offers a more convenient and practical approach to realize an enhanced and stable SC for many applications, such as real-time, ultrafast and singleshot spectroscopic measurements and high speed optical switching and signal processing in optical communication. We also demonstrate the utility of the present technique in the context of optical parametric amplification (OPA), in which the amplified signal quality showed significant improvement in the $\mathrm{CW}$-triggered broadband pumping case versus the untriggered case.

\section{EXPERIMENTAL SETUP}

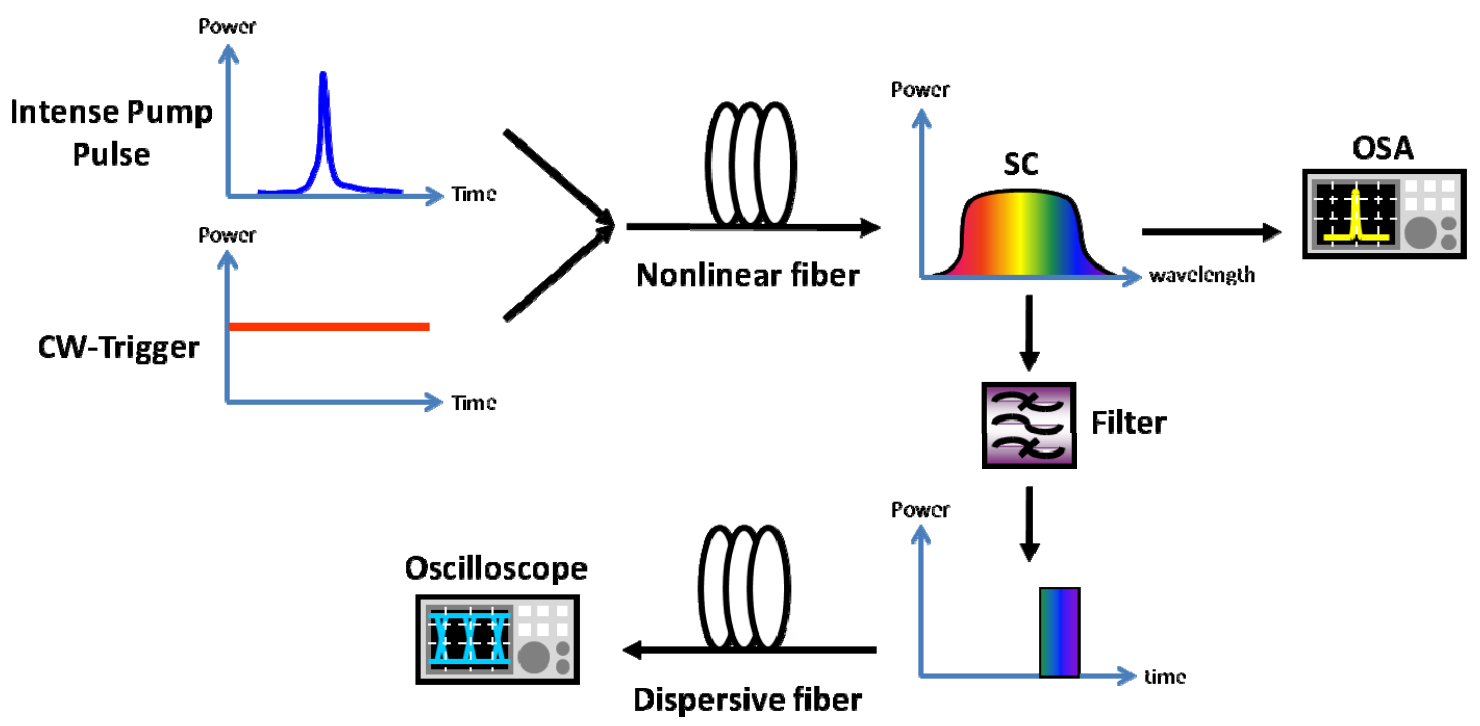

Figure 1. Schematic diagram of the experimental setup generating the CW-triggered SC.

Figure 1 shows the schematic of the experimental setup for generating the minute-CW-triggered SC. Intense pump pulses are delivered by a picosecond mode-locked fiber laser (with repetition rate of 78-MHz, pulse width of 5.8- 
ps and peak power at $22 \mathrm{~W}$ ) and are launched into a 50-m highly nonlinear dispersive fiber (HNL-DSF) (with zerodispersion wavelength (ZDW) at $1554 \mathrm{~nm}$, dispersion slope of $0.035 \mathrm{ps} / \mathrm{nm}^{2} / \mathrm{km}$ and nonlinear coefficient of $14 \mathrm{~W}^{-1} \mathrm{~km}^{-1}$ ) to generate the SC. The center pump wavelength was $1554.5 \mathrm{~nm}$ which lies in the anomalous dispersion regime. On the other hand, we employed a tunable $\mathrm{CW}$ laser source with a power of $\sim 80 \mu \mathrm{W}$ as the weak $\mathrm{CW}$ trigger which is also coupled into the HNL-DSF and co-propagate with the pump in order to observe the enhancement effect. A bandpass filter with a bandwidth of $10 \mathrm{~nm}$ was used to select the long-wavelength $\mathrm{SC}$ spectral region for further investigation, such as the real-time pulse-amplitude statistical measurements. The filtered SC output was dispersed by $200-\mathrm{m}$ dispersion-compensating fiber (DCF) with dispersion of $18.5 \mathrm{ps} / \mathrm{nm}$ before detected by a real-time oscilloscope with a bandwidth and a sample rate of $4 \mathrm{GHz}$ and $20 \mathrm{GSa} / \mathrm{s}$, respectively. It is also possible to measure the individual pulse energy directly using a photodetector and a sampling oscilloscope while real-time spectral measurements are not required [11].

\section{EXPERIMENTAL RESULTS AND DISCUSSIONS}

\subsection{Output SC power enhancement by the CW-trigger}
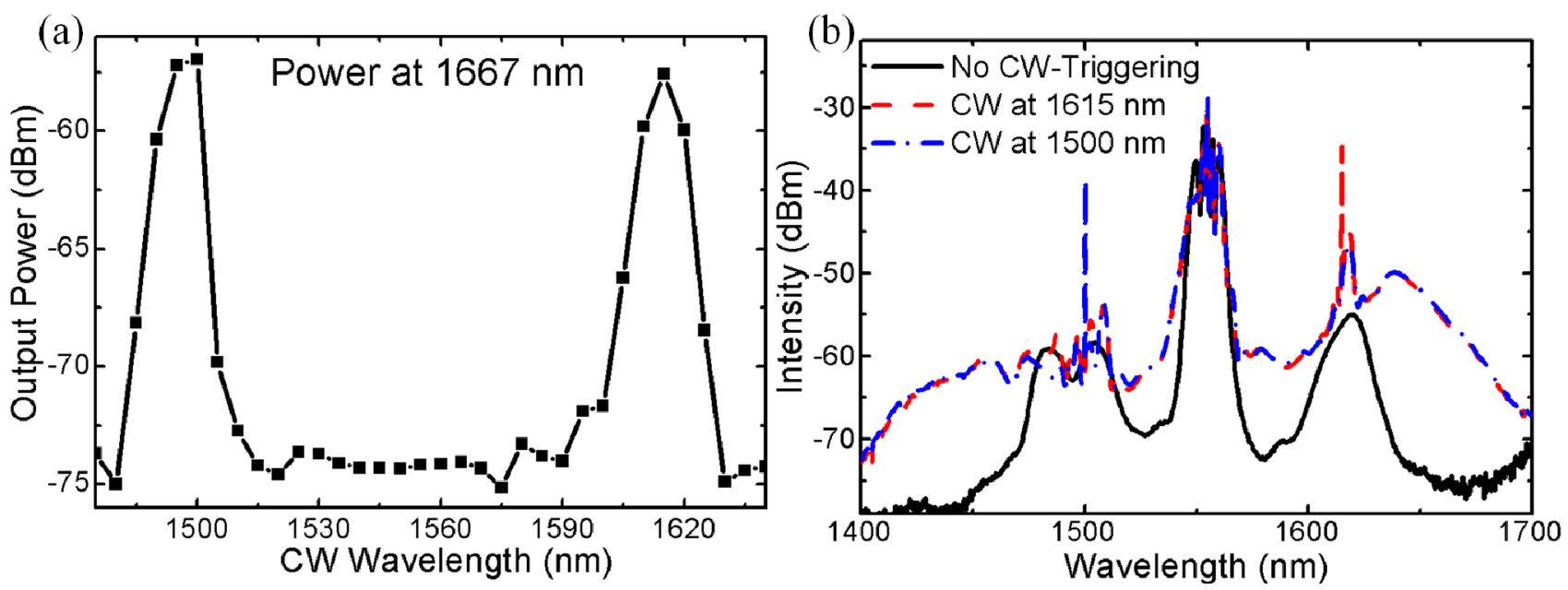

Figure 2(a). SC enhancement as the function of CW-trigger's wavelength. The peak power of the pump pulse at $22 \mathrm{~W}$ whereas the power of the SC trigger is $0.08 \mathrm{~mW}$. (b) Untriggered SC spectrum (black line), SC spectrum with the $\mathrm{CW}$ trigger at at $1505 \mathrm{~nm}$ (redline) and $\mathrm{SC}$ spectrum with the $\mathrm{CW}$ trigger at $1614 \mathrm{~m}$ (blue line).

Figure 2(a) depicts the SC enhancement as a function of the $\mathrm{CW}$ trigger wavelengths. From the figure, it can be clearly observed that the enhancement effect manifests itself in the two main lobes, which roughly symmetrically straddled the pump wavelength, resembling the MI gain spectrum. The pronounced enhancement only occurs when the weak CW trigger has the wavelength match with the MI shift or in the other word, within the MI gain bandwidth - a region where the weak trigger experiences large MI gain. When the cumulative MI gain is sizable enough, soliton fission emerges and leads to substantial spectral broadening subsequently. The trigger wavelengths at $1505 \mathrm{~nm}$ and $1614 \mathrm{~nm}$ are at the peaks of the lobes and both give the greatest SC enhancement.

We also investigate how the SC spectrum can be affected by the minute $\mathrm{CW}$ trigger. Figure 2(b) compares the $\mathrm{CW}$-triggered and untriggered SC spectra. It is clearly observed that the SC spectrum is significantly modified and enhanced by an extremely weak CW trigger ( $>200,000 \times$ weaker than pump). Notably, the CW trigger introduces $\sim 20-$ $25 \mathrm{~dB}$ increase in power on both the red-shifted $(\sim 1400 \mathrm{~nm}$ range $)$ and blue-shifted $(\sim 1700 \mathrm{~nm}$ range $)$ sides of the SC spectrum. The enhanced SC spectra spans from $<1400 \mathrm{~nm}$ to $>1700 \mathrm{~nm}$, much wider than the untriggered SC which only has the bandwidth of $\sim 200 \mathrm{~nm}$, i.e. from $\sim 1450 \mathrm{~nm}$ to $1650 \mathrm{~nm}$. Interestingly, under the identical pump condition, the 
enhanced SC spectrum with a trigger at $1614 \mathrm{~nm}$ is remarkably similar to the one with a $\mathrm{CW}$ trigger at another wavelength of $1505 \mathrm{~nm}$, in terms of the spectral shape and the undulation characteristics.

The results shown in figure 2 are significant that the enhanced SC can be realized by simply turning on a weak $\mathrm{CW}$ trigger, with a properly chosen wavelength within the lobe regions. Prior works showed that similar active control of $\mathrm{SC}$ can be realized by using a pulse-seeding mechanism or intensity modulation of the pump pulses at a frequency in the $\mathrm{THz}$ regime. Nevertheless, it is mandatory for the pulse-seeding scheme to maintain highly precise time-delay adjustment (down to ps) between the seed and the pump. While direct optical modulation technology at $\mathrm{THz}$ frequency is not readily available, special wavelength conversion techniques [8] are required to achieve the aforementioned modulation-enhanced SC. In contrast, the fact that the present $\mathrm{CW}$-triggering technique requires only the wavelength tuning to achieve the enhanced SC with similar quality renders itself a much simpler approach to utilize the enhanced SC in a more practical way.
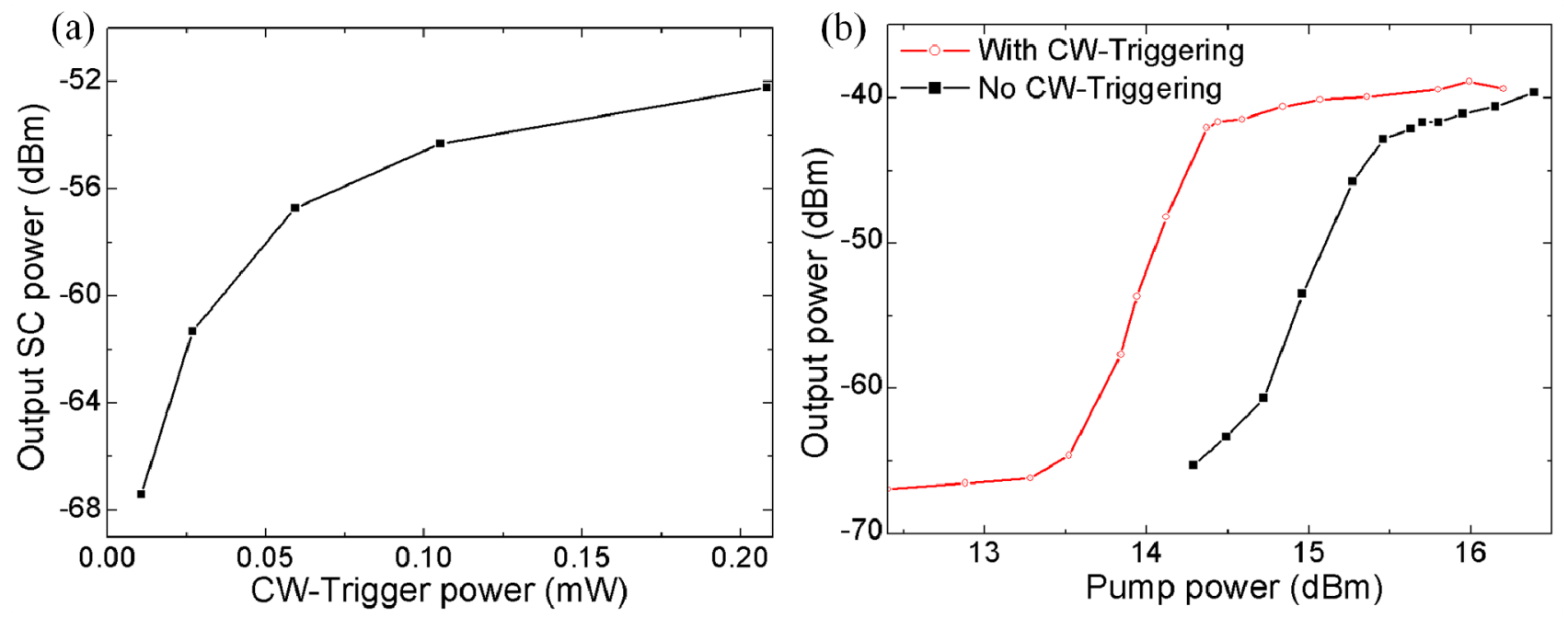

Figure 3(a). Output SC power as the function of CW trigger power at a fixed pump power. The CW-trigger wavelength is $1667 \mathrm{~nm}$. (b)

Filtered SC power as the function of pump power. Red-circle line represents the CW-triggered case while the black-square line represents the untriggered case.

Figure 3 (a) shows the SC output power (at $1667 \mathrm{~nm}$ ) as the function of the power of the CW-trigger (at 1615 $\mathrm{nm})$. We observe the output SC power enhancement essentially scales with the CW-trigger power except that such enhancement starts to roll-off for further increase in the CW-trigger's power .Moreover, we filter a portion of SC spectrum $(1640 \mathrm{~nm}-1650 \mathrm{~nm})$ and measured the output SC power at that wavelength band as the function of the pump power (Fig. 3(a) and (b)). A threshold feature can be observed in both the untriggered SC and CW-triggered SC cases. Such threshold indicates the onset of the soliton fission in the SC generation process [3]. From Fig. 3(b), we can show that introducing the $\mathrm{CW}$-trigger can effectively reduce the threshold pump power - making a more power-efficient SC generation process.

\subsection{Coherence properties of stimulated SC}

We study the SC amplitude statistics in both $\mathrm{CW}$-triggered and untriggered SC situations using real-time wavelength-to-time mapping technique [12]. This technique has been employed for identifying ultrafast and rare event, such as capturing rogue waves in real-time [4], high-speed spectroscopy [13] and imaging [5]. Figure 4 shows the power histogram of 1562 filtered SC pulses $(1640 \mathrm{~nm}-1650 \mathrm{~nm})$. It shows a clear long-tail distribution - a signature of the extreme-value statistics, resembling the key feature of the optical rogue waves [4]. However, when a weak CW at 1614 $\mathrm{nm}$ is added, it alters the SC amplitude statistics to an almost Gaussian distribution, and shows a $\sim 60 \%$ reduction in the standard deviation. We attribute the observed pulse-amplitude variation to the relative intensity noise (RIN) and the finite coherence length (and hence the linewidth) of the CW source. Clearly, RIN can disturb the initial condition of soliton fission and thus deteriorates the SC stability. On the other hand, the coherence length of the $\mathrm{CW}$ source has to be 
long enough compared to the fiber length in order to preserve the phase coherence. In our case, the coherence length of the $\mathrm{CW}$ source is $\sim 30 \mathrm{~m}$ compared to the fiber length of $\sim 50 \mathrm{~m}$. A narrow-linewidth $\mathrm{CW}$ source with low RIN is hence essential for maintaining high stability and coherence in the CW-triggered SC.

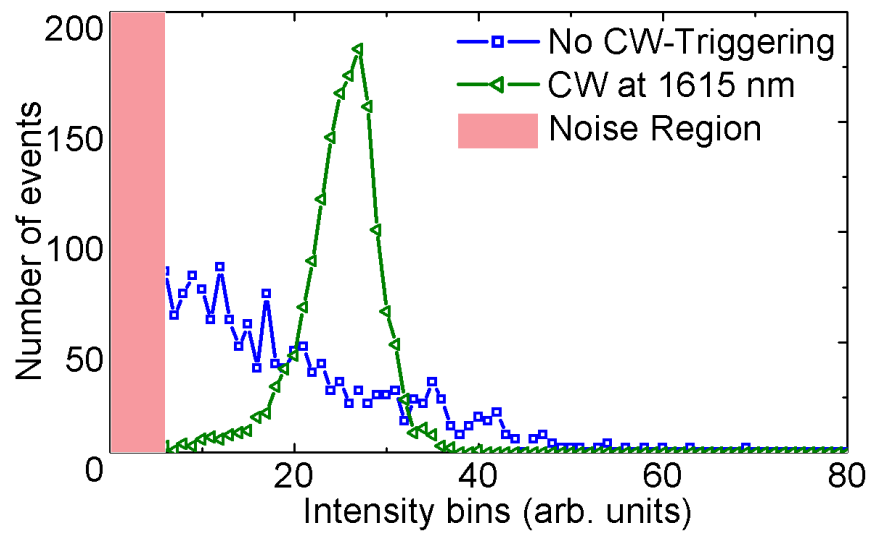

Figure 4. Filtered pulse-to-pulse amplitude histograms of the untriggered SC (blue) and the CW-triggered SC (green). The pump power is adjusted in order to compare the statistics under similar SC average power level. The red shaded region represents the noise floor of the measurement.

We further investigate the coherent properties of the SC based on the interferometric measurements. The filtered SC pulses are sent to an interferometer with unbalanced arm lengths. The coherence is evaluated by observing the interference fringes in the SC spectrum when consecutive coherent pulses are overlapped with a temporal delay. We quantify the coherence of the SC by a quantity called fringe visibility which is given by [14]:

$$
V=\left(I_{\max }-I_{\min }\right) /\left(I_{\max }+I_{\min }\right)
$$

where $I_{\max }$ and $I_{\min }$ corresponds the maximum and mininmum intensities of the fringes, respectively. For the untriggered $\mathrm{SC}$, the MI growth starts spontaneously from noise and hence the SC is in general less coherent, especially in the redshifted spectral regions. In contrast, when the SC is triggered by a weak CW light, pulse-to-pulse coherence is improved as shown in high visibility (Figure 5).

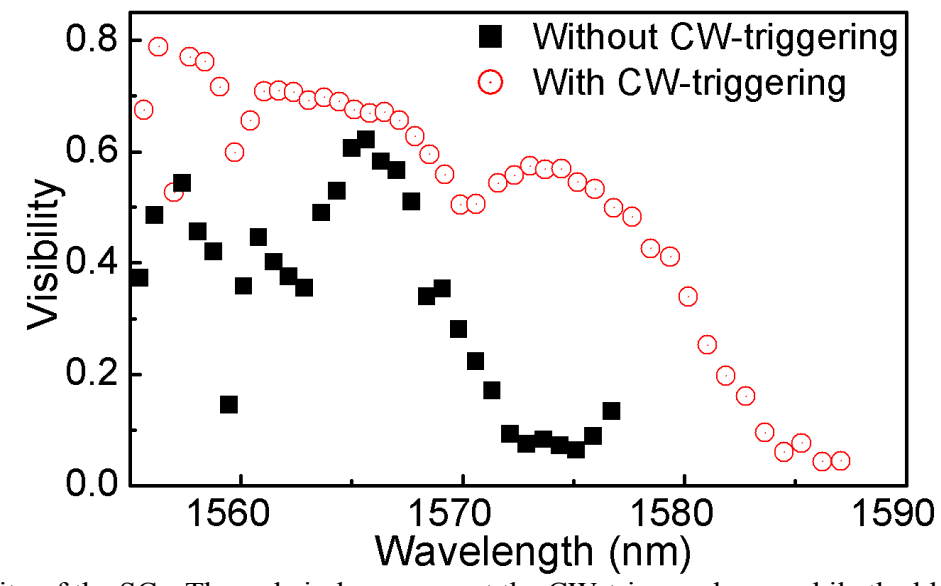

Figure 5. Fringe visibility of the SCs. The red circles represent the CW-triggered case while the black squares represent the untriggered case.

\subsection{CW-triggered SC as a broadband pump for optical parametric amplifier}




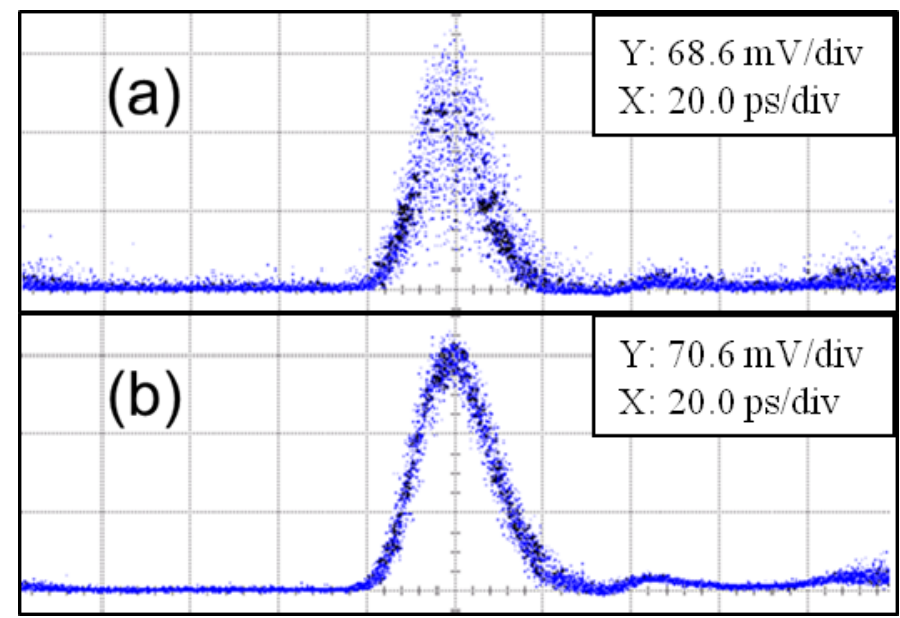

Figure 6. Output amplified signals of the FOPA (a) without CW-triggering. (b) with CW-triggering. The chirped pump wavelength range is $1561 \mathrm{~nm}-1566 \mathrm{~nm}$ and the signal wavelength is $1554.5 \mathrm{~nm}$.

The technique presented here will be applicable to in many applications, ranging from optical signal processing to high-speed biomedical diagnostics. As an example of the utility of the $\mathrm{CW}$-triggered SC, we consider widebandpumped optical parametric amplification (OPA). OPA is a nonlinear optical effect based on the third-order Kerr susceptibility of the material [15]. It has been recognized to be a versatile optical amplification approach in fiber-optics communication because of its ultra-wide gain bandwidth and flexibly-tunable gain spectrum [16]. In general, the amplified signal quality in OPA is critically related to that of the input pump pulse [16]. We employed a portion (1561 $\mathrm{nm}-1566 \mathrm{~nm}$ ) of the SC as the pump. The filtered pump is then temporally-chirped by a spool of dispersive fiber [17]. Such chirped pump pulse was then amplified and combined with a weak pulse signal at $1554.5 \mathrm{~nm}$ in a nonlinear fiber where OPA is taken place. Figure 6 clearly shows that the output amplified signal quality is greatly improved, in terms of intensity noise and timing-jitter, when the broadband pump is CW-triggered. When the broadband pump is CW-triggered, the timing jitter of the amplified signal is reduced from 6 ps to $1.1 \mathrm{ps}$. The timing jitter of the amplified signal with the CW-triggered SC is primarily attributed to group velocity dispersion (GVD) during the chirping of the pump pulse in a dispersive fiber. Such improvement in timing jitter and intensity noise by the $\mathrm{CW}$-triggering scheme is of great importance to maintain the signal quality in all-optical signal processing applications.

\section{CONCLUSION}

In conclusion, we demonstrate an enhanced $\mathrm{SC}$ generation by introducing a minute $\mathrm{CW}$ trigger. The $\mathrm{CW}$ triggered SC shows considerable spectral broadening ( $>100 \mathrm{~nm}$ wider), enhancement in output intensity $(>20 \mathrm{~dB})$, and improvement in SC coherence. The present $\mathrm{CW}$-triggering scheme only requires the trigger-wavelength tuning for optimization and thus makes it as an attractive and simple tool for realizing stable and enhanced SC, with a wide range of input pump conditions, namely from the shorter-pulse $(\sim \mathrm{fs})$ to the longer-pulse $(>\mathrm{ps})$ pumping schemes, in order to generate a more stable and enhanced SC - expanding the scope of possibilities in SC generation, especially for the applications in which single-shot, real-time spectroscopic measurements and imaging is required.

\section{ACKNOWLEDGEMENT}

The work in this paper is partially supported by grants from the Research Grants Council of the Hong Kong Special Administrative Region, China (Project No. HKU 7179/08E, HKU 7183/09E, and HKU 717510E). The authors would also like to acknowledge Sumitomo Electric Industries for providing the HNL-DSF and Alnair Laboratories for providing the variable bandwidth tunable bandpass filter. 


\section{REFERENCES}

[1] R. R. Alfano, ed., “The Supercontinuum Laser Source,” (Springer, 2006).

[2] J. M. Dudley, G. Genty and S. Coen, "Supercontinuum generation in photonic crystal fiber," Rev. Mod. Phys., 78, 1135 (2006).

[3] G. Genty, S. Coen and J. M. Dudley, "Fiber supercontinuum sources,"J. Opt. Sco. Am. B., 24, 1771 (2007).

[4] D. R. Solli, C. Ropers, P. Koonath and B. Jalali, "Optical rouge waves," Nature (London)., 450, 1054 (2007).

[5] K. Goda, K. K. Tsia and B. Jalali, "Serial time-encoded amplified imaging for real-time observation of fast dynamic phenomena," Nature. 458, 1145 (2009).

[6] D. L. Marks, A. L. Oldenburg, J. J. Reylonds, "Study of an ultrahigh-numerical-aperture fiber continuum generation source for optical coherence tomography," Opt. Lett., 27, 2010 (2002).

[7] D. R. Solli, C. Ropers, P. Koonath and B. Jalali, "Active Control Rogue Waves for Stimulated Supercontinuum Generation,” Phys. Rev. Lett., 101, 233902 (2008).

[8] G. Gently, J. Dudley and B. Eggleton, "Modulation control and spectral shaping of optical fiber supercontinuum generation in the picosecond regime," Appl. Phys. B: Lasers Opt., 94, 187 (2009).

[9] D. Wildanger, E. Rittweger, L. Kastrup, and S.W. Hell, "STED microscopy with a supercontinuum laser source,"Opt. Express, 16, 9614 (2008).

[10] A. Gambetta, V. Kumar, G. Grancini, D. Polli, R. Ramponi, G. Cerullo, and M. Marangoni, "Fiber-format stimulated-Raman-scattering microscopy from a single laser oscillator," Opt. Lett., 35, 226 (2010)

[11] C. Lafargue, J. Bolger, G. Genty, F. Dias, J. M. Dudley and B. J. Eggleton, "Direct detection of optical rogue wave energy statistics in supercontinuum generation," Electron. Lett., 45, 217 (2009).

[12] T. Jannson, "Real-time Fourier transformation in dispersive optical fibers," Opt. Lett., 8, 232 (1983).

[13]D. R. Solli, J. Chou and B. Jalali, "Amplified wavelength-time transformation for real-time spectroscopy," Nature Photon., 2, 48 (2008).

[14] M. Born, E. Wolf, ed., "Principle of Optics, ” (Cambridge University Press, 1999).

[15] G. P. Agrawal, "Nonlinear Fiber Optics", 4th ed., Elsevier (Singapore) Pte Ltd, (2009).

[16]M. E. Marhic, "Fiber Optical Parametric Amplifiers, Oscillators and Related Devices", 1st ed., (Cambridge University Press, 2008).

[17] C. Zhang, K. K. Y. Cheung, K. K. Tsia, and K. K. Y. Wong, "Fiber optical parametric with broadband chirped pump," IEEE Photon. Technol. Lett., (to be submitted).

*Email: tsia@hku.hk 